\title{
Exact solutions of nonlinear PDE, nonlinear transformations and reduction nonlinear PDE to a quadrature
}

\author{
Yang Lei, Liu Jianbin, Yang Kongqing \\ Department of physics, Lanzhou University, Gansu 730000, China
}

\begin{abstract}
A method to construct the exact solution of the PDE is presents, which combines the two kind methods(the nonlinear transformation and RQ(Reduction the PDE to a Quadrature problem) method).The nonlinear diffusion equation is chosen to illustrate the method and the exact solutions are obtained.
\end{abstract}

Seeking the exact solutions of the nonlinear PDE(Partial Differential Equation) play an important role in the nonlinear problems, a number of methods have been developed, such as, Inverse Scattering theory[1], but in some cases $[2,3]$, these methods cannot do very well. So the simple and direct methods to find analytic solutions of PDE have drawn a lot of interest, for example, Hirota's bilinear methods[4], the truncated Painlevè expansion[5,6], homogeneous balance method[7,8], reduction the PDE to a quadrature problem[9], the hyperbolic tangent function-series method[10] and etc.

In the sense of the seeking solutions, these direct methods can be divided into two kinds, one kind bases on the nonlinear transformation, such as, Hirota's bilinear methods bases on the Hirota transformation, by using the transformation the nonlinear PDE becomes the bilinear forms, then seeking the solution of the bilinear forms leads to obtain the solution of the origin PDE. The truncated Painlevè expansion bases on the Painlevè expansion, by using the leading-order analysis a set of Painlevé-Bäcklund equations are obtained, then seeking the solution of the set of Painlevè-Bäcklund equations leads to obtain the solution of the origin PDE. The homogeneous balance method gives the nonlinear transformation based on a simple program, by using the transformation a set of equations are obtained, then seeking the solution of the set of equations leads to obtain the solution of the origin PDE. And the special nonlinear transformation can be given for the special nonlinear PDE.

Another kind of method bases on the direct ansatz of the solution, most of those methods are applied to seek the travelling wave solution, such as, the hyperbolic tangent function-series method and Reduction the PDE to a Quadrature problem(RQ method). In generalized, the nonlinear PDE is written as

$$
P\left(u, u_{x}, u_{t}, u_{x x}, u_{x t}, u_{t t}, \cdots \cdots\right)=0 .
$$

Introducing the similarity variable $\xi=x-v t$, the travelling wave solutions $u(\xi)=u(x-v t)$ satisfy

$$
P^{\prime}\left(u, u_{\xi}, u_{\xi \xi}, \cdots \cdots\right)=0
$$

The hyperbolic tangent function-series method makes the ansatz,

$$
u(\xi)=\sum_{i=0}^{m} a_{i}(\tanh (b \xi))^{i}
$$

where the velocity $v$, the integer $m$, the real $a_{i}$ and $b$ are parameter to be determined, inserting the ansatz into the equation(2), a set of the nonlinear algebra equations of these undetermined parameters are obtained, solving it, the solution of the equation(1) is obtained. Reduction the PDE to a Quadrature problem(RQ method) is an interesting method for looking for the travelling wave solutions, the essence of the method can be presented in this way: First, giving a ansatz for $\frac{d}{d x} u(x, t)$, namely,

$$
u_{\xi}=\sum_{i=0}^{N} a_{i} u^{b_{i}}(\xi),
$$

where $a_{i}, b_{i}$ are undetermined constants(usually $N \leq 3$ ), substituting the formula (4) into the equation (2), when the suitable constants $a_{i}, b_{i}$ are chosen, the equation (2) becomes a identical equation. Then solving the equation (2) becomes to solve the equation (4), it's obviously the solution of the equation (4) can be obtained just by integration.

This paper try to combine the two kinds of methods to solve the nonlinear PDE. The first step, the origin PDE is transformed into other equations by a nonlinear transformation, then RQ method is applied to these equations to obtain the exact solution. Here 1+1 D nonlinear diffusion equation is chosen,

$$
u_{t}-u_{x x}+P(u)=0
$$


to illustrate this method, where $u$ is a real scalar field defined over the spatial $x$ and temporal $t, P(u)=\sum_{i=0}^{d} a_{i} u^{i}$ is a polynomial of $u$, for most of physical problems require $d \leq 5$, we choose

$$
P(u)=\sum_{i=0}^{d} a_{i} u^{i}=a_{0}+a_{1} u+a_{2} u^{2}+a_{3} u^{3}+a_{4} u^{4}+a_{5} u^{5},
$$

where $a_{i}$ are parameters. When $a_{0}=a_{3}=a_{4}=a_{5}=0$, the equation(5) becomes the Fisher equation. When $a_{0}=a_{2}=a_{4}=a_{5}=0$, the equation(5) becomes the Newell-Whitehead equation (also known as the KolmogorovPetrovsky-Piskunov equation). This paper is organized as follows. In Sec II, the fisher equation is discussed, combining the truncated Painlevè expansion and the RQ method, the exact solution of the fisher equation is obtained. In Sec III, the equation $u_{t}-u_{x x}+a_{1} u+a_{2} u^{2}+a_{3} u^{3}+a_{4} u^{4}=0$ and equation $u_{t}-u_{x x}+a_{0}+a_{1} u+a_{4} u^{4}=0$ are discussed, combines a special transformation for the equation and the RQ method, the exact solutions of these equations are obtained. In Sec IV, the fifth order the NW(KPP) equation is discussed, combines a special transformation for the NW equation and the RQ method, the exact solution of the NW equation is obtained. In Sec V, the main conclusions are listed.

1. In this section, the Fisher equation is discussed, which is

$$
u_{t}-u_{x x}+a u+b u^{2}=0
$$

Taking the truncated Painlevé expansion,

$$
u(x, t)=f(x, t)^{-I} \sum_{l=0}^{I} u(x, t)_{l} f(x, t)^{l},
$$

where $I$ is a natural number to be determined, $u(x, t)_{l}$ is analytic functions. The leading-order analysis for the equation(7) yields $I=2$, so that

$$
u(x, t)=\frac{u_{2}(x, t)}{f(x, t)^{2}}+\frac{u_{1}(x, t)}{f(x, t)}+u_{0}(x, t) .
$$

Substituting (9) into (7) with Mathematica, making the coefficients of like powers of vanish, so as to obtain the following set of Painlevé-Bäcklund equations,

$$
\begin{aligned}
& f^{-1}: a u_{1}+2 b u_{0} u_{1}+u_{1 t}-u_{1 x x}=0 \\
& f^{-2}: b u_{1}^{2}+a u_{2}+2 b u_{0} u_{2}-u_{1} f_{t}+u_{2 t}+2 f_{x} u_{1 x}+u_{1} f_{x x}-u_{2 x x}=0 \\
& f^{-3}: b u_{1} u_{2}-u_{2} f_{t}-u_{1} f_{x}^{2}+2 f_{x} u_{2 x}+u_{2} f_{x x}=0 \\
& f^{-4}: b u_{2}-6 f_{x}^{2}=0 .
\end{aligned}
$$

Here $u_{0}(x, t)$ satisfied

$$
u_{0 t}-u_{0 x x}+a_{1} u_{0}+a_{2} u_{0}^{2}=0
$$

which has the similar from of the equation (7), thus the set of the equations (9)-(11) constitutes an auto-Bäcklund transformation. It's obviously, the equations(11) have a trivial solution $u_{0}(x, t)=0$. Based on the trivial solution, introducing the similarity variable $\xi=x-v t$, and taking the following ansatz

$$
f^{\prime}(\xi)=\alpha f(\xi)+\beta
$$

where $\alpha$ and $\beta$ are undetermined parameters. Substituting (12) into the equations (10), choosing $a=6 \alpha^{2}, v=-5 \alpha$, the equations (10) become identical equations, then the equation(12) can be solved just by integration. So the solution of the equation $(7)$ is

$$
u(x, t)=-\frac{6 c e^{\alpha x} \alpha^{3}\left(c \alpha e^{\alpha x}-2 \beta e^{5 \alpha^{2} t}\right)}{b\left(c \alpha e^{\alpha x}-\beta e^{5 \alpha^{2} t}\right)} .
$$

2. In this section, the equation $u_{t}-u_{x x}+a_{1} u+a_{2} u^{2}+a_{3} u^{3}+a_{4} u^{4}=0$ and equation $u_{t}-u_{x x}+a_{0}+a_{1} u+a_{4} u^{4}=0$ are discussed. The first equation is given 


$$
u_{t}-u_{x x}+a u+b u^{2}+c u^{3}+d u^{4}=0 .
$$

A transformation for equation(14) is introduced

$$
u(x, t)=\frac{g(x, t)}{f(x, t)^{\frac{2}{3}}}-u_{0}(x, t),
$$

substituting the formula(15) into the equation(14) with Mathematica, make the coefficients of like powers of $f(x, t)$ vanish, so as to obtain the following set equations,

$$
\begin{aligned}
& f^{-\frac{2}{3}}: a g-2 b g u_{0}+3 c g u_{0}^{2}-4 d g u_{0}^{3}+g_{t}-g_{x x}=0, \\
& f^{-\frac{4}{3}}: b-3 c u_{0}+6 d u_{0}^{2}=0 ; \\
& f^{-\frac{5}{3}}: g f_{t}-2 g_{x} f_{x}-g f_{x x}=0 ; \\
& f^{-\frac{6}{3}}: c-4 d u_{0}=0 ; \\
& f^{-\frac{8}{3}}: 9 d g^{3}-10 f_{x}^{2}=0
\end{aligned}
$$

and $u_{0}(x, t)$ satisfied the equation

$$
u_{0 t}-u_{0 x x}+a u_{0}+b u_{0}^{2}+c u_{0}^{3}+d u_{0}^{4}=0,
$$

which has the similar from of the equation (14), thus the set of the equations (15)-(17) constitutes an auto-Bäcklund transformation. It's obviously, the equations $(17)$ have a trivial solution $u_{0}(x, t)=0$. Based on the trivial solution, introducing the similarity variable $\xi=x-v t$, and taking the following ansatz

$$
f^{\prime}(\xi)=\alpha f(\xi)+\beta f(\xi)^{2},
$$

where $\alpha$ and $\beta$ are undetermined parameters. Substituting (18) into the equations (16), choosing $\alpha=\frac{3 c^{\frac{3}{2}}}{8 \sqrt{10} d}, b=\frac{3 c^{2}}{8 d}$, $a=\frac{3 c^{3}}{64 d^{2}}, v=-\frac{7 \alpha}{3}$. The equations (16) become identical equations, then the equation(18) can be solved just by integration. So the solution of the equation (14) is

$$
u=\frac{4\left(\frac{30}{d}\right)^{\frac{1}{3}} d\left(\frac{\alpha^{2} e^{\alpha x+\alpha v t+c_{1}}}{\left(e^{c_{1}}-e^{\alpha(x+v t)} \beta\right)^{2}}\right)^{\frac{2}{3}}-3 c\left(\frac{\alpha e^{\alpha(x+v t)}}{e^{c_{1}-e^{\alpha(x+v t)} \beta}}\right)^{\frac{2}{3}}}{12 d\left(\frac{\alpha e^{\alpha(x+v t)}}{e^{c_{1}-e^{\alpha(x+v t)} \beta}}\right)^{\frac{2}{3}}} .
$$

The second equation is

$$
u_{t}-u_{x x}+a u+d u^{4}=0
$$

the transformation (15) is workable for the equation(20). The auto-Bäcklund transformation can be obtained similarly. Based on the trivial solution $u_{0}(x, t)=0$, introducing the similarity variable $\xi=x-v t$, and taking the following ansatz

$$
f^{\prime}(\xi)=\alpha+\beta f(\xi)
$$

where $\alpha$ and $\beta$ are undetermined parameters. Choosing $a=-\frac{10 \beta^{2}}{9}, v=\frac{7 \beta}{3}$, then the solution of the equation (20) is

$$
u=\frac{10^{\frac{1}{3}}}{d}\left(\frac{c_{1} e^{\beta\left(x+\frac{7 \beta t}{3}\right)} \beta}{3 c_{1} e^{\beta\left(x+\frac{7 \beta t}{3}\right)}-\frac{3 \alpha}{\beta}}\right)^{\frac{2}{3}} .
$$

3. The fifth order the NW(KPP) equation is discussed as follow:

$$
u_{t}-u_{x x}+a u+c u^{3}+e u^{5}=0 .
$$

A transformation for equation(23) is introduced

$$
u(x, t)=\frac{g(x, t)}{\sqrt{f(x, t)}}+u_{0}(x, t),
$$


substituting the formula(24) into the equation(23) with Mathematica, make the coefficients of like powers of $f(x, t)$ vanish, so as to obtain the following set equations,

$$
\begin{aligned}
& f^{-\frac{1}{2}}: a g+3 c g u_{0}^{2}+5 e g u_{0}^{4}+g_{t}-g_{x x}=0, \\
& f^{-1}: 3 c+10 e u_{0}^{2}=0, \\
& f^{-\frac{3}{2}}: 2 c g^{3}+20 e g^{3} u_{0}^{2}-g f_{t}+2 f_{x} g_{x}+g f_{x x}=0, \\
& f^{-2}: 5 e g^{4} u_{0}=0 \\
& f^{-\frac{5}{2}}: 4 e g^{4}-3 f_{x}^{2}=0 .
\end{aligned}
$$

and $u_{0}(x, t)$ satisfied the equation

$$
u_{0 t}-u_{0 x x}+a u_{0}+c u_{0}^{3}+e u_{0}^{5}=0,
$$

which has the similar from of the equation (23), thus the set of the equations (24)-(26) constitutes an auto-Bäcklund transformation. It's obviously, the equations $(26)$ have a trivial solution $u_{0}(x, t)=0$. Based on the trivial solution, introducing the similarity variable $\xi=x-v t$, and taking the following ansatz

$$
f^{\prime}(\xi)=\alpha f(\xi)+\beta f(\xi)^{2},
$$

where $\alpha$ and $\beta$ are undetermined parameters. Substituting (27) into the equations (25), choosing

$$
\begin{aligned}
& v=\frac{1}{3}\left(2 \sqrt{3} \sqrt{-4 a+\frac{c^{2}}{e}}-\sqrt{3} c \sqrt{\left.\frac{1}{e}\right),}\right. \\
& \alpha=\frac{1}{2}\left(-\sqrt{3} c \sqrt{\frac{1}{e}}-v\right),
\end{aligned}
$$

the equations (25) become identical equations, then the equation(27) can be solved just by integration. So the solution of the equation $(23)$ is

$$
u(x, t)=\left(\frac{3}{4 e}\right)^{\frac{1}{4}} \sqrt{\frac{\alpha e^{c_{1}}}{e^{c_{1}}-e^{\alpha(x+v t)} \beta}} .
$$

In conclusion, a method to construct the exact solution of the PDE is presented in this paper, which combine the two kind methods(the nonlinear transformation and RQ method). The $1+1 \mathrm{D}$ nonlinear diffusion equation are discussed as example to show this method. When the parameter $a_{i}$ in the equation(6) take four kinds of value, the four equations are discussed. For every equation, the auto-Bäcklund transformation of it is presented.

Acknowledgments: This work was supported by the National Natural Science Foundation of China. The corresponding email address: yangkq@lzu.edu.cn (Yang Kongqing).

[1] C.S.Gardner, J.M.Greene, M.D.Kruskal, R.M.Miura, Phys.Rev.Lett., 19(1067), 1095.

[2] M.J.Skrinjar, D.V.Kapor, S.D.Stojanovic, J.Phys.Condens.Matter, 32(1989), 725.

[3] Nozaki.K, J.Phys.Soc.Japan, 56(1987), 3052.

[4] R.Hirota, J.Math.Phys, 14(1973), 810.

[5] J.Weiss, M.Tabor, G.Carnevale, J.Math.Phys, 24 (1983), 522.

[6] F.Cariello, M.Tabor, Physica D,53 (1991), 59.

[7] M.Wang, Phys.Lett.A, 216 (1995), 67.

[8] L.Yang, Z.Zhu,Y.Wang, Phys.Lett.A, 260 (1999), 55

[9] M.Otwinowski, al, Phys.Lett.A, 128 (1988), 483.

[10] L.Huibin, W.Kelin, J.Phys.Math.Gen, 23(1990), 3923. 